\title{
Intravascular migration of a previously functioning epidural catheter
}

\author{
Jooyeon Jeon, In Ho Lee, Hea-Jo Yoon, Myoung-Goo Kim, and Pil-Moo Lee \\ Department of Anesthesiology and Pain Medicine, Cheil General Hospital, Kwandong Uiversity Medical School, Seoul, Korea
}

The use of regional anesthesia for spontaneous delivery and cesarean section has significantly increased, due to both patient demand and the reduced risk of maternal mortality and morbidity compared with general anesthesia [1]. Despite the benefits, inadvertent intravascular and intrathecal injection of a local anesthetic may cause severe complications, including systemic toxicity, total spinal anesthesia, and a high spinal block.

We report a case of late migration of a previously functioning epidural catheter into an epidural vein and the local anesthetic systemic toxicity in a pregnant woman.

A 36 -year-old woman (height $=162 \mathrm{~cm}$, weight $=65 \mathrm{~kg}$ ) at 40 weeks gestation was admitted to a delivery ward during labor. There was nothing significant in her medical history except for an appendectomy and a breast fibroadenoma excision. Results of laboratory testing, chest X-ray, and electrocardiogram were within the normal range. She requested epidural analgesia during labor.

Epidural analgesia was administered without difficulty using a loss of resistance technique. The epidural space was identified using an 18 G Tuohy needle (Perifix; B-Braun, Melsungen, Germany) at a depth of $4 \mathrm{~cm}$ at the L3-L4 interspace, and a 20 G multi-orifice epidural catheter (Perifix) was then threaded easily. There was no bleeding during the procedure, and an aspiration test was negative. A $3 \mathrm{ml}$ test dose of $0.2 \%$ ropivacaine was administered via the epidural catheter, and the maternal heart rate and blood pressure did not change significantly for three minutes. There was no tinnitus, perioral tingling, or motor block. A $9 \mathrm{ml}$ bolus dose of $0.2 \%$ ropivacaine was administered, and a continuous infusion pump $(0.2 \%$ ropivacaine $27 \mathrm{ml}$ mixed with fentanyl $150 \mu \mathrm{g}$ and normal saline $60 \mathrm{ml}$ ) was started at a rate of $10 \mathrm{ml} / \mathrm{hr}$. Pain was sufficiently relieved after continuous infusion, and she did well without the motor block. The highest level of sensory block was verified at the T10 level using cold sensation.

After three hours of labor, the patient was scheduled for emergent cesarean delivery due to fetal distress. The patient was brought into the operating room, moved herself to the operating table, and was placed supine with left uterine displacement. Initial blood pressure was 135/80 $\mathrm{mmHg}$, and heart rate was $85 \mathrm{bpm}$. A $16 \mathrm{ml}$ mixed solution of Lidocaine (2\% lidocaine $12 \mathrm{ml}$ mixed with fentanyl $100 \mu \mathrm{g}, 1: 200,000$ epinephrine $50 \mu \mathrm{g}$, sodium bicarbonate $2 \mathrm{mEq}$ : total dose 16 $\mathrm{ml}$ ) was administered slowly via the epidural catheter without an aspiration test or an epinephrine-containing epidural test dose. Within one minute, the patient reported perioral tingling and blurred vision. She became drowsy and did not respond to the anesthesiologist's voice. Her heart rate increased from 85 to $100 \mathrm{bpm}$, and blood pressure increased from 120/80 to 140/90 mmHg. Immediately we suspected intravascular injection of local anesthetic. An aspiration test was performed, and bloodstained fluid was aspirated. A rapid sequence intubation was immediately performed with propofol $100 \mathrm{mg}$ and succinylcholine $50 \mathrm{mg}$. Rocuronium $20 \mathrm{mg}$ was administered and anesthesia was maintained with $2 \mathrm{~L} / \mathrm{min} \mathrm{O}_{2}, 2 \mathrm{~L} / \mathrm{min}_{2} \mathrm{O}$, and $1 \%$ sevoflurane. The patient's vital signs were stabilized. A $3.8 \mathrm{~kg}$ baby was delivered ten minutes after the first incision. Oxytocin at $20 \mathrm{IU}$ was administered after the delivery. Apgar

Corresponding author: Hea-Jo Yoon, M.D., Department of Anesthesiology and Pain Medicine, Cheil General Hospital, Kwandong Uiversity Medical School, 1-19, Mukjeong-dong, Jung-gu, Seoul 100-380, Korea. Tel: 82-2-2000-7822, Fax: 82-2-2000-7477, E-mail: heajo7890@hanmail.net (c) This is an open-access article distributed under the terms of the Creative Commons Attribution Non-Commercial License (http:// creativecommons.org/licenses/by-nc/3.0/), which permits unrestricted non-commercial use, distribution, and reproduction in any medium, provided the original work is properly cited. 
scores were 7 and 9 at 1 and 5 minutes, respectively. Atropine 0.5 $\mathrm{mg}$ and neostigmine $1 \mathrm{mg}$ were administered for the reversal of muscle relaxation. The epidural catheter was removed before extubation. The patient was then extubated uneventfully and transferred to a recovery room.

The patient was comfortable, and vital signs were stable in the recovery room. No neurological signs were observed for over one hour. Furthermore, no motor disturbances were noted. The patient was transferred to a ward.

Epidural analgesia provides good quality analgesia for labor pain. However, unintentional and unrecognized cannulation of an epidural vein or migration of the catheter may cause serious complications. The reported incidence in pregnant patients is about $15 \%$, higher than non-pregnant patients due to markedly dilated epidural veins [2]. Pan et al. [2] reported 6\% initial vascular cannulation of epidural catheters and $0.25 \%$ incidence of late migration of epidural catheters into the vascular space in a large retrospective analysis of almost 15,000 regional anesthetic procedures during labor or cesarean section.

In this case, epidural analgesia was performed without difficulty, and no blood was aspirated. The highest level of sensory block was verified at the T10 level using cold sensation. The patient's pain was controlled with epidural analgesia, however she became drowsy and did not respond to the anesthesiologist's voice after the injection of a local anesthetic in the operation room. Blood-stained fluid was then aspirated from the catheter.

In this case, a previously functioning epidural catheter migrated into an epidural vessel.

The inherent mobility of women during labor may contribute to epidural catheter movement and migration [3]. When the catheter migrates inward, cannulation of intravascular, subdural, or subarachnoid spaces is possible.

There are several ways to avoid systemic toxicity, including careful aspiration of the epidural catheter, fractionation of epidural dose, and the use of an epinephrine-containing epidural test dose [4]. Aspiration is frequently used to confirm correct placement of an epidural catheter. Single orifice catheters should not be tested using aspiration as more than $50 \%$ false negative results have been noted [4]. In contrast, multi-orifice catheters can be tested using aspiration. It is prudent to aspirate the catheter prior to each epidural dose.

Performing the epinephrine-containing epidural test in pregnancy is controversial because of decreased autonomic nerve system response to epinephrine, difficult interpretation due to heart rate increase during uterine contractions, and the possibility of decreased uterine blood supply [5].

Our case describes the migration of a previously functioning epidural catheter into an epidural vessel. We think that this migration can be explained by patient posture changes and movements. In conclusion, the aspiration test and epinephrinecontaining test dose should always be performed prior to a local epidural anesthetic for cesarean section even if the function of the epidural catheter was previously established. Careful observation of neurologic signs is also important.

\section{References}

1. Morgan BM, Aulakh JM, Barker JP, Reginald PW, Goroszeniuk T, Trojanowski A. Anaesthetic morbidity following caesarean section under epidural or general anaesthesia. Lancet 1984; 1: 328-30.

2. Pan PH, Bogard TD, Owen MD. Incidence and characteristics of failures in obstetric neuraxial analgesia and anesthesia: a retrospective analysis of 19,259 deliveries. Int J Obstet Anesth 2004; 13: 227-33.

3. Crosby ET. Epidural catheter migration during labour: a hypothesis for inadequate analgesia. Can J Anaesth 1990; 37: 789-93

4. Bell DN, Leslie K. Detection of intravascular epidural catheter placement: a review. Anaesth Intensive Care 2007; 35: 335-41.

5. Marcus MA, Gogarten W, Vertommen JD, Buerkle H, Van Aken H. Haemodynamic effects of repeated epidural test-doses of adrenaline in the chronic maternal-fetal sheep preparation. Eur J Anaesthesiol 1998; 15: 320-3. 